\title{
Construcción de una Escala de Dominio Moral en Adolescentes
}

\author{
Construction of a Moral Domain Scale in Adolescents
}

\author{
Rubén Andrés Miranda-Rodríguez ${ }^{1}$ y Mirna García-Méndez ${ }^{2}$
}

\begin{abstract}
Resumen
El objetivo fue identificar cómo se expresa y estructura el dominio moral desde la teoría de los fundamentos morales en una muestra de adolescentes mexicanos. Se elaboró una escala de dominio moral para adolescentes y se identificó cómo se relaciona con la teoría de los fundamentos morales mediante tres estudios: análisis de contenido, análisis factorial exploratorio y análisis factorial confirmatorio aunado a su correlación con el cuestionario de los fundamentos morales. Los resultados revelan cinco principios morales para la muestra de adolescentes estudiada: amor/respeto, lealtad/familia, ofensa, equidad y perjuicio Se sugiere que estos principios son conceptual y estructuralmente diferentes que los fundamentos morales universales. Se argumentan las diferencias conceptuales y estructurales y se discute en favor de considerar la cultura local, así como la etapa del desarrollo para evaluar el dominio moral.
\end{abstract}

Palabras clave: dominio moral, adolescencia, desarrollo moral, fundamentos morales, cultura

\begin{abstract}
We aimed to identify how moral domain is expressed and structured from the moral foundations theory in a sample of Mexican adolescents. A scale of moral domain for adolescents was elaborated and it was identified how it relates to the moral foundations theory through three studies: content analysis, exploratory factor analysis and confirmatory factor analysis together with its correlation with the moral foundations' questionnaire. The results show five moral principles for the sample of adolescents studied: love/respect, damage, equity, loyalty/family and offense. We suggest that these principles are conceptually and structurally different from universal moral foundations. The conceptual and structural differences are argued and discussed towards considering the local culture, as well as the stage of human development to evaluate the moral domain.
\end{abstract}

Keywords: moral domain, adolescence, moral development, moral foundations, culture

\footnotetext{
${ }^{1}$ Doctorante en psicología social. Universidad Nacional Autónoma de México, Facultad de Estudios Superiores Zaragoza. Av. Guelatao No. 66 (Eje 7 Oriente). Colonia Ejército de Oriente. Ciudad de México. C.P.09230, México. Tel.: 0445518190776. Correo: r.andresmiranda.r@gmail.com

${ }^{2}$ Doctora en Psicología Social, Profesora Titular Tiempo Completo. Universidad Nacional Autónoma de México, Facultad de Estudios Superiores Zaragoza. Av. Guelatao No. 66 (Eje 7 Oriente). Colonia Ejército de Oriente. Ciudad de México. C.P.09230. Edificio de Gobierno Planta alta, Carrera de Psicología. Tel.: 56230553. Correo: mina@unam.mx

Revista Iberoamericana de Diagnóstico y Evaluación - e Avaliação Psicológica. RIDEP · No53 · Vol.4 · 169-183 · 2019

ISSN: 1135-3848 print /2183-6051online
} 


\section{Introducción}

La adolescencia es una etapa que contiene cambios psicosociales y biológicos que afectan la dirección y las características del desarrollo humano debido a que se adquieren nuevos roles sociales y responsabilidades en lo escolar, familiar y social (Aguilar, Valencia, \& Sarmiento, 2007). En esta etapa se involucran procesos como identidad, sexualidad, intimidad y autonomía (Góngora \& Castro-Solano, 2014). Sin embargo, también se suelen observar comportamientos que contravienen las normas y las reglas establecidas, e.g. agresión, bullyng o delincuencia (Kollerová, Janošová, \& Říčan, 2014; Mansilla, 2000; Sanabria \& Uribe 2009).

Con relación a los comportamientos problemáticos de los adolescentes, se realizó un estudio de factores de riesgo y victimización en adolescentes que cometieron delitos de alto impacto en México (Reinserta Un Mexicano A.C., 2018). Se encontró que en los adolescentes recluidos en diferentes centros de detención del país en 2018 , el $39.8 \%$ de los hombres y el 33.3 $\%$ de las mujeres fueron reincidentes en delitos de robo, homicidio o secuestro. El $40 \%$ de estos reincidentes cometieron su primer delito a los 13 años.

Este tipo de conductas cuestiona y demanda la conformación apropiada de una educación moral enfocada en esta etapa del desarrollo (Garaigordobil, 2005; Pozzoli, Gini, \& Thornberg, 2016). En México, se argumenta que esta educación se debe centrar en lo cívico, donde se promuevan principios morales y de participación ciudadana (Conde-Flores, García-Cabrero, \& Alba-Meraz, 2017) con énfasis en que los principios morales se puedan reconocer a través de la evaluación psicológica (Kollerová et al., 2014). De tal manera que es importante identificar los principios que se deben promover en la educación de los adolescentes, por lo que se sugiere partir del constructo de dominio moral, definido como el conjunto de principios sobre el bien y el mal que regulan el comportamiento de las personas (Graham et al., 2011; Turiel, 1983).

El dominio moral parte de la perspectiva filosófica del realismo científico, en el que los principios morales se definen como afirmaciones normativas sobre lo que se debe y no se debe hacer, con la característica de que se desarrollan a través de la experiencia, se expresan cognoscitivamente y pueden medirse como hechos psicológicos (Railton, 1986). Esta perspectiva da pauta a la teoría de los fundamentos morales, como una propuesta de principios identificados científicamente. Los fundamentos morales se definen como principios sustentados en sistemas intuitivos, innatos y culturalmente variables en su relevancia, es decir, la teoría sugiere que estos fundamentos se utilizan en todas las culturas, pero para una sociedad un fundamento es más importante que otro para otra sociedad. Los fundamentos morales son: Cuidado/Daño, Justicia/injusticia, Lealtad/ Traición, Autoridad/Subversión y Pureza/ Degradación (Haidt \& Joseph, 2004, 2007).

El fundamentos de cuidado/daño modera los sentimientos de compasión por las personas vulnerables y las que sufren, la justicia/injusticia involucra las necesidades de reciprocidad en las relaciones sociales, la lealtad/traición se refiere al deber de mantener la cohesión y el valor al grupo de pertenencia, la autoridad/subversión comprende el respeto que se le da a la jerarquía en la organización social y la pureza/degradación incluye la distinción de lo desagradable en función de rituales y reglas de santidad (Graham et al., 2011; Graham et al., 2013). Para dar sustento a la naturaleza innata de estos principios, la teoría de los fundamentos morales se remite a explicaciones con orientación evolutiva y predisposición biológica. Es decir, una perspectiva de la moralidad como herramienta del ser humano para su adaptación y supervivencia como especie (De Waal, 1996; Pinker, 2002).

Uno de los cuestionamientos a la teoría de los fundamentos morales se refiere a la relación naturaleza-cultura (Haste, 2013). Se menciona que la estructura de los fundamentos morales muestra variaciones asociadas al contexto en donde se aplica (Berniūnas, Dranseika, \& Sousa, 2016). Resultado de estas observaciones, se resalta la posibilidad de asumir varios dominios morales dependientes de cómo se entienden y se expresan en cada cultura, en lugar de un solo dominio moral universal y biológicamente predispuesto (Lees \& Gino, 2017). Por lo que es importante entender la moralidad como la conjunción de las necesidades biológicas y los procesos 
socioculturales con los que interactúan las personas a lo largo de su desarrollo y que conforman su noción del bien y del mal. Se toma en consideración que la variabilidad cultural de los fundamentos morales no sólo se aplica a su relevancia, sino también a su contenido conceptual y uso del discurso (Haste, 2013).

Con base en las críticas y sugerencias hacia el estudio de los fundamentos morales, es relevante explorar el cómo las personas se expresan y le dan significado a estos principios desde su cultura (Harré, 2002). Particularmente en la cultura mexicana, Díaz-Guerrero $(1994 ; 2003)$ refiere que es necesario reconocer cómo se manifiestan sus reglas y patrones de comportamiento a través de las normas, creencias, valores y tradiciones, que se pueden identificar en las formas de expresión de los mexicanos sobre qué se debe hacer y cómo se deben comportar las personas. Es así que se toma una perspectiva que considere el impacto que tienen los significados socioculturales en la orientación moral de los pensamientos y conductas de los mexicanos (Díaz-Loving, 2011), de manera particular en los adolescentes.

Se sugiere la construcción de un instrumento que contenga la conceptuación que los adolescentes tienen de los principios mencionados en la teoría de los fundamentos morales (Graham et al., 2011). El objetivo es aportar elementos que puedan ser empleados en la de educación moral y participación cívica de los adolescentes, a través del reconocimiento de los principios propios de la cultura a la que pertenecen (Haste, Bermúdez, \& Carretero, 2017). Esto con la finalidad de colaborar en la prevención de comportamientos antisociales o delictivos, ya que se ha demostrado que los adolescentes con mayores desacuerdos o desconexión con los principios morales son susceptibles a este tipo de comportamientos (Doyle \& Bussey, 2018; Leenders \& Brugman, 2005; Palmer \& Hollin, 1998; Pozzoli et al., 2016).

Con el propósito de elaborar un instrumento sobre dominio moral en adolescentes valido y confiable, esta investigación se dividió en tres estudios: en el primero se empleó un análisis de contenido, en el segundo un análisis factorial exploratorio y en el tercero un análisis factorial confirmatorio. Así mismo, se obtuvieron las correlaciones de la escala de dominio moral con el cuestionario de los fundamentos morales (Graham et al., 2011).

\section{Estudio 1}

Para identificar el dominio moral con base en una perspectiva sociocultural, es importante interpretar el discurso en su contexto cultural con la finalidad de precisar el conocimiento referente a cómo piensan y se comportan las personas moralmente (Barrios, Barbato, \& Branco, 2012; Kwok \& Selman, 2017). Así, surge la necesidad de identificar el contenido conceptual que los adolescentes les dan a los componentes del dominio moral, objetivo de este estudio, en el que se utilizará la técnica de análisis de contenido.

\section{Método}

\section{Participantes}

Participaron 100 adolescentes inscritos en secundarias o preparatorias públicas y laicas (51 $\%$ mujeres y $49 \%$ hombres) con un rango de edad de 12 a 17 años $(M=14.90, D E=1.44)$ residentes de la Ciudad de México y municipios del Estado de México, con el criterio de que no tuvieran reportes de comportamiento antisocial o delictivo por parte de las autoridades de su escuela. El muestreo fue no probabilístico intencional.

\section{Instrumento}

Cuestionario de preguntas abiertas exprofeso (Anexo 1). Se redactaron doce preguntas abiertas con base en la literatura de dominio moral sustentada en la teoría de los fundamentos morales (Graham et al., 2011; Graham et al., 2013). Se separaron los conceptos en positivos y negativos debido a que pueden no formar parte de un continuo (Wiltermuth, Monin, \& Chow, 2010). Se agregaron al cuestionario los estímulos "moralidad" e "inmoralidad", con la finalidad de reconocer de manera global la percepción de lo bueno y lo malo para los adolescentes (Wojciszke, 2005).

\section{Procedimiento}

Para obtener los datos se acudió a diferentes secundarias y preparatorias, se solicitaron permisos a las autoridades correspondientes de las escuelas, se explicaron los objetivos de la 
investigación y cada autoridad firmó un documento de consentimiento informado después de revisar los instrumentos. Las aplicaciones fueron de manera grupal en los salones de clase, con la supervisión de los investigadores y una autoridad respectiva encargada de cada grupo, como profesores o tutores. Así mismo, cada adolescente contaba con un asentimiento de participación en el que tenía la libertad de decidir si deseaba o no contestar el cuestionario. Se garantizó su anonimato y confidencialidad. Después de cada aplicación, se agradeció a estudiantes y autoridades su colaboración y se proporcionaron datos de contacto para cualquier aclaración. Los datos se transcribieron con ayuda del programa Excel, en el que se organizaron y analizaron las respuestas.

\section{Resultados}

Con base en las respuestas a las doce preguntas abiertas, se realizó un análisis de contenido por categorías deductivas, es decir, conformadas a priori del análisis y con base en la teoría. Este tipo de análisis es recomendado porque considera categorías establecidas neutralmente y centradas en la teoría (Mayring, 2014). Así como los fundamentos morales se consideran inicialmente intuitivos e innatos, se espera que sean comprensibles universalmente (Graham et al., 2013), y culturalmente variables no sólo en su relevancia sino también en su expresión discursiva (Haste, 2013).

Cada categoría representó un fundamento moral separado en positivo y negativo como se mencionó en la sección de instrumentos. Se definieron a partir de las palabras obtenidas con mayor frecuencia en las respuestas de los adolescentes. En la Tabla 1 se muestran los resultados del análisis de contenido, donde se observa que las categorías comparten indicadores, de tal modo que los adolescentes utilizaron palabras como respeto o maltrato para responder más de una pregunta. Estos resultados fueron la base para redactar los reactivos del estudio 2 .

\section{Estudio 2}

Algunos instrumentos miden el desarrollo moral sobre la base de un dominio enfocado en principios de cuidado y justicia (Gibbs et al., 1984; Kohlberg, 1987). Actualmente, con la adhesión de los principios de lealtad, autoridad y pureza (Graham et al., 2011; Shweder, Mahapatra, \& Miller, 1987), se han elaborado instrumentos que se centran en puntajes obtenidos por muestras conformadas por adultos y sobre la base de los cinco fundamentos morales sin considerar la variabilidad cultural antes mencionada (Clifford, Iyengar, Cabeza, \& Sinnott-Amstrong, 2015; Graham et al., 2011). Con el propósito de contar con una escala válida y confiable que evalúe los principios que conforman el dominio moral en adolescentes, en este estudio se tuvo como objetivo elaborar un instrumento a partir de los resultados del estudio $1 \mathrm{y}$ con los planteamientos de la teoría de los fundamentos morales (Graham et al., 2013).

\section{Método}

\section{Participantes}

Participaron 747 adolescentes inscritos en secundarias o preparatorias públicas y laicas (54.6 $\%$ mujeres y $45.4 \%$ hombres) con un rango de edad de 12 a 17 años $(M=14.52, D E=1.41)$, residentes de la Ciudad de México y municipios del Estado de México, sin reportes de comportamiento antisocial o delictivo por parte de las autoridades de su escuela. El muestreo fue no probabilístico intencional.

\section{Instrumento}

Escala de dominio moral para adolescentes. Con los resultados del estudio 1 se redactaron 78 reactivos en formato Likert con seis opciones de respuesta (1=totalmente en desacuerdo y $6=$ totalmente de acuerdo) que evalúan los fundamentos morales.

\section{Procedimiento}

Se acudió a secundarias y preparatorias de la ciudad de México. Para obtener los permisos de las autoridades correspondientes, su consentimiento y el asentimiento de los adolescentes, se siguió el procedimiento descrito en el estudio 1. Las aplicaciones fueron de manera grupal en los salones de clase, con la supervisión de los investigadores y una autoridad respectiva 
Tabla 1. Análisis de contenido del dominio moral en adolescentes $(\mathrm{N}=100)$

\begin{tabular}{|c|c|c|c|}
\hline Categoría & Definición & $\begin{array}{l}\text { Indicadores más frecuentes } \\
\text { (ejemplos) }\end{array}$ & $\begin{array}{c}\text { Total de } \\
\text { indicadores }\end{array}$ \\
\hline Cuidado & $\begin{array}{l}\text { Muestras de respeto y afecto a través de acciones } \\
\text { de apoyo y unión. }\end{array}$ & $\begin{array}{l}\text { Respeto, } \\
\text { convivencia/unión, } \\
\text { ayuda/apoyo }\end{array}$ & 52 \\
\hline Justicia & $\begin{array}{l}\text { Búsqueda de trato igualitario y respeto a los } \\
\text { derechos de todos sin importar condiciones } \\
\text { sociales. }\end{array}$ & $\begin{array}{l}\text { Igualdad, respeto, trato humano, } \\
\text { beneficio, equidad, derechos. }\end{array}$ & 53 \\
\hline Lealtad & $\begin{array}{l}\text { Demostración de confianza y fidelidad en } \\
\text { relaciones cercanas como la amistad o la familia. }\end{array}$ & $\begin{array}{l}\text { Confianza, fidelidad, no traicionar, } \\
\text { apoyolayuda, amistad, respeto. }\end{array}$ & 51 \\
\hline Autoridad & $\begin{array}{l}\text { Reconocimiento a una jerarquía responsable, } \\
\text { justa y que busca el bienestar social. }\end{array}$ & $\begin{array}{l}\text { Bien común, justa, respeto, } \\
\text { responsabilidad, deber, honestidad. }\end{array}$ & 49 \\
\hline Pureza & $\begin{array}{l}\text { Preservación de la honestidad y las buenas } \\
\text { intenciones a través del ejercicio de los valores. }\end{array}$ & $\begin{array}{l}\text { Bondad, sin malas intenciones, } \\
\text { honestidad, lealtad, respeto. }\end{array}$ & 51 \\
\hline Moralidad & $\begin{array}{l}\text { Valoración de acciones fundamentadas en } \\
\text { principios y normas sobre lo correcto. }\end{array}$ & $\begin{array}{l}\text { Bondad, valores, respeto, no } \\
\text { maldad, honestidad, hacer lo } \\
\text { correcto. }\end{array}$ & 39 \\
\hline Daño & $\begin{array}{l}\text { Provocación de problemas y maltratos que } \\
\text { perjudiquen directamente a otros. }\end{array}$ & $\begin{array}{lcr}\text { Maltrato } & \text { físico, } & \text { maltrato } \\
\text { psicológico, } & \text { problema, } & \text { baja } \\
\text { autoestima. } & \end{array}$ & 59 \\
\hline Injusticia & $\begin{array}{l}\text { Acciones que promueven la desigualdad y el } \\
\text { perjuicio a la vulnerabilidad de otros. }\end{array}$ & $\begin{array}{l}\text { Maltrato, daño, falta de respeto, } \\
\text { beneficio, desigualdad, culpa. }\end{array}$ & 48 \\
\hline Traición & $\begin{array}{l}\text { Faltas a la confianza y los acuerdos establecidos } \\
\text { en relaciones cercanas. }\end{array}$ & $\begin{array}{l}\text { Confianza, mentira, engaño, } \\
\text { amistad, no guardar secretos. }\end{array}$ & 47 \\
\hline Subversión & $\begin{array}{l}\text { Justificación basada en faltas de una autoridad } \\
\text { para elegir desobedecerla. }\end{array}$ & $\begin{array}{l}\text { Injusticia, maltrato, desacuerdo, } \\
\text { derechos, su propio beneficio. }\end{array}$ & 49 \\
\hline Degradación & $\begin{array}{l}\text { Pérdida de la bondad y la honestidad a través de } \\
\text { acciones consideradas incorrectas. }\end{array}$ & $\begin{array}{l}\text { Malicia, } \quad \text { mentiras, } \quad \text { traición, } \\
\text { groserías, daño, drogas, alcohol. }\end{array}$ & 43 \\
\hline Inmoralidad & $\begin{array}{l}\text { Acciones que fallan a las normas y los valores } \\
\text { sociales sobre lo correcto. }\end{array}$ & $\begin{array}{l}\text { Maldad, falta de respeto, no } \\
\text { valores, daño, no ser bueno, } \\
\text { mentiras. }\end{array}$ & 40 \\
\hline
\end{tabular}

encargada de cada grupo, como profesores o tutores. Se hizo énfasis en el anonimato y confidencialidad de la información. Los datos recabados se analizaron en el programa SPSS versión 24.

\section{Resultados}

Aunque los 78 reactivos mostraron índices adecuados de discriminación $(p<.01)$ se eliminaron dos por presentar correlaciones bajas con los demás reactivos. Con los 76 reactivos se realizó un análisis factorial exploratorio con el método de factorización por ejes principales con rotación oblicua oblimin, ya que se consideran recomendables para la validez de constructo debido a que considera las correlaciones entre factores (Abad, Olea, Ponsoda, \& García, 2011; Ferrando \& Anguiano-Carrasco, 2010), además este método de rotación se ha utilizado para la medición de los principios morales en función de que tienden a estar correlacionados significativamente entre sí (Graham et al., 2011).
Se eligieron los reactivos con cargas factoriales mayores a .40 para la conformación de los factores, por lo que se seleccionaron 20 reactivos distribuidos en cinco factores. Las propiedades psicométricas de la escala se muestran en la Tabla 2.

En la Tabla 3 se presentan las correlaciones entre los factores de la escala de dominio moral para adolescentes.

De acuerdo con los resultados, se propone definir el dominio moral en adolescentes como un proceso culturalmente variable, donde se manifiestan principios sobre el bien y el mal que dirigen las relaciones sociales hacia la preservación del amor, el respeto mutuo, la lealtad a la familia y la equidad, así como el rechazo a las ofensas como faltas de respeto a los valores y a las acciones perjudiciales e injustas. En la Tabla 4 se muestran las definiciones de los factores. 
Tabla 2. Análisis factorial exploratorio de la Escala de Dominio Moral para Adolescentes (N=747)

\begin{tabular}{|c|c|c|c|c|c|c|}
\hline $\begin{array}{l}\text { 69. Tratar a las personas con respeto ayuda a ser una } \\
\text { sociedad más justa. }\end{array}$ & .670 & .282 & .308 & .304 & .330 & \\
\hline $\begin{array}{l}\text { 73. Procurar el bienestar de aquellos que amamos es lo más } \\
\text { importante. }\end{array}$ & .669 & .304 & .142 & .284 & .316 & \\
\hline $\begin{array}{l}\text { 78. El respeto es el camino necesario para ser una mejor } \\
\text { sociedad. }\end{array}$ & .633 & .202 & .382 & .334 & .258 & \\
\hline $\begin{array}{l}\text { 74. Es necesaria una autoridad que se preocupe por el } \\
\text { bienestar de todos. }\end{array}$ & .618 & .246 & .248 & .384 & .313 & \\
\hline $\begin{array}{l}\text { 63. Cuidar a otras personas es una manera de expresar lo } \\
\text { valiosas que son. }\end{array}$ & .611 & .392 & .169 & .280 & .350 & \\
\hline $\begin{array}{l}\text { 44. Las personas siempre deben ser leales a los miembros } \\
\text { de su familia. }\end{array}$ & .311 & .740 & .367 & .120 & .249 & \\
\hline 16. La lealtad a la familia es lo más importante de todo. & .339 & .739 & .258 & .176 & .178 & \\
\hline $\begin{array}{l}\text { 28. El respeto es necesario para jurar lealtad a los seres } \\
\text { queridos. }\end{array}$ & .308 & .593 & .338 & .214 & .153 & \\
\hline $\begin{array}{l}\text { 56. La falta de respeto sólo lleva a la gente a malas } \\
\text { consecuencias. }\end{array}$ & .364 & .377 & .685 & .274 & .373 & \\
\hline $\begin{array}{l}\text { 25. La mentira es un camino que sólo lleva a malas } \\
\text { consecuencias. }\end{array}$ & .293 & .354 & .666 & .241 & .356 & \\
\hline 55. La venganza es la peor forma de buscar justicia. & .198 & .250 & .665 & .204 & .307 & \\
\hline $\begin{array}{l}\text { 17. Todos tenemos derecho a que nuestra opinión sea } \\
\text { tomada en cuenta. }\end{array}$ & .339 & .282 & .266 & .609 & .296 & \\
\hline $\begin{array}{l}\text { 27. La ley se debe aplicar a todos por igual, sin importar si } \\
\text { son ricos o pobres. }\end{array}$ & .225 & .164 & .177 & .523 & .240 & \\
\hline 4. La equidad de género es un valor indispensable. & .219 & -.041 & .166 & .480 & .253 & \\
\hline $\begin{array}{l}\text { 8. Todas las personas tienen los mismos derechos, sin } \\
\text { importar su preferencia sexual. }\end{array}$ & .249 & .011 & -.076 & .469 & -.102 & \\
\hline $\begin{array}{l}\text { 50. Es indignante que algunas personas se beneficien a } \\
\text { costa del esfuerzo de otros. }\end{array}$ & .186 & .171 & .364 & .264 & .753 & \\
\hline $\begin{array}{l}\text { 41. Es horrible que una persona le provoque traumas } \\
\text { emocionales a otra. }\end{array}$ & .337 & .166 & .222 & .362 & .677 & \\
\hline 48. Pienso que es inaceptable dañar a otras personas. & .337 & -.207 & .386 & .335 & .641 & \\
\hline $\begin{array}{l}\text { 31. Es injusto que una sola persona se quede todo el crédito } \\
\text { de un logro que se obtuvo en equipo. }\end{array}$ & .237 & -.045 & .225 & .210 & .589 & \\
\hline $\begin{array}{l}\text { 43. Una persona que le guste perjudicar a los demás es un } \\
\text { ser desagradable. }\end{array}$ & .354 & .296 & .289 & .228 & .589 & \\
\hline Número de reactivos & 5 & 3 & 3 & 4 & 5 & 20 \\
\hline Varianza explicada & 25.501 & 6.457 & 5.069 & 3.358 & 2.687 & 43.073 \\
\hline Alfa de Cronbach & .757 & .704 & .624 & .633 & .714 & .844 \\
\hline KMO & & & & & & .874 \\
\hline
\end{tabular}

Nota. Los números en negrita representan las cargas factoriales más altas.

Tabla 3. Correlación de factores de la Escala de Dominio Moral para Adolescentes $(\mathrm{N}=747)$

\begin{tabular}{lccccc}
\hline & Amor/Respeto & Lealtad/Familia & Ofensa & Equidad & Perjuicio \\
\hline Amor/Respeto & 1 & & & & \\
Lealtad/Familia & $.445^{* *}$ & 1 & & & \\
Ofensa & $.386^{* *}$ & $.414^{* *}$ & 1 & & \\
Equidad & $.364^{* *}$ & $.334^{* *}$ & $.284^{* *}$ & 1 & $.385^{* *}$ \\
Perjuicio & $.445^{* *}$ & $.214^{* *}$ & $.387^{* *}$ & 1 \\
\hline
\end{tabular}

** Correlación estadísticamente significativa al .01.

\section{Estudio 3}

Los resultados del estudio 2 muestran una estructura factorial basada en un contenido conceptual propio de la adolescencia, que se refleja en una conceptuación de un dominio moral perteneciente a esta etapa. Para corroborar los resultados, se llevó a cabo un análisis factorial confirmatorio, en el que se deben hacer hipótesis a priori sobre el número de factores, lascorrelaciones entre éstos, cómo saturan las variables observadas 
Tabla 4. Definiciones de los factores de la Escala de Dominio Moral para Adolescentes

\begin{tabular}{|c|c|c|}
\hline & Factor & Definición \\
\hline 1. & Amor/Respeto & $\begin{array}{l}\text { Juicios que favorecen las muestras de afecto, la preocupación por el bienestar del otro y } \\
\text { la demostración de que las personas son valiosas. }\end{array}$ \\
\hline 2. & Lealtad/Familia & $\begin{array}{l}\text { Juicios que fortalecen la estabilidad de los preceptos familiares y el valor de la familia } \\
\text { como guía moral. }\end{array}$ \\
\hline 3. & Ofensa & $\begin{array}{l}\text { Juicios que reprueban la deshonestidad y la pérdida de valores a través de actos como la } \\
\text { mentira y la venganza. }\end{array}$ \\
\hline 5. & Perjuicio & $\begin{array}{l}\text { Juicios que reprueban la voluntad de agredir, provocar dolor y aprovecharse de las } \\
\text { condiciones de otros. }\end{array}$ \\
\hline
\end{tabular}

en ellos y si existen correlaciones entre los términos de error o específicos (Abad et al., 2011). Este estudio se realizó con una muestra diferente de los estudios 1 y 2 con la hipótesis de que se mantendrán los cinco factores correlacionados entre sí, con saturaciones de las variables observadas (los reactivos) correspondientes a su factor latente y sin la presencia de correlaciones entre errores. Además, se realizaron correlaciones con los factores del cuestionario de los fundamentos morales (Graham et al., 2011) traducido al español (Bedregal, León, Shand, \& Mosso, 2013), con la finalidad de reconocer las convergencias y divergencias de la escala de dominio moral para adolescentes.

\section{Método}

\section{Participantes}

Participaron 410 adolescentes inscritos en secundarias o preparatorias públicas y laicas (57.6 $\%$ mujeres y $42.4 \%$ hombres) con un rango de edad de 12 a 17 años $(M=14.66, D E=1.31)$, residentes de delegaciones de la Ciudad de México y municipios del Estado de México, sin reportes de comportamiento antisocial o delictivo por parte de las autoridades de su escuela. El muestreo fue no probabilístico intencional.

\section{Instrumentos}

Escala de dominio moral para adolescentes (Anexo 2). Descrita en el estudio 2.

Cuestionario de los fundamentos morales. Se utilizó el instrumento de la teoría de los fundamentos morales (Graham et al., 2011) traducido al español (Bedregal et al., 2013), compuesto por 30 reactivos, divididos en dos secciones: 15 reactivos en términos de relevancia y 15 reactivos en términos de estar de acuerdo o en desacuerdo. Es una escala Likert con seis intervalos de respuesta $(0=$ nada relevante en la primera sección y totalmente en desacuerdo en la segunda y $5=$ muy relevante en la primera sección $\mathrm{y}$ totalmente de acuerdo en la segunda). Los reactivos de ambas secciones en conjunto se distribuyen en cinco factores que corresponden con los cinco fundamentos morales: cuidado/daño $(\alpha=.69)$, justicia/injusticia $(\alpha=.65)$, lealtad/traición $(\alpha=.71), \quad$ autoridad/subversión $\quad(\alpha=.74) \quad y$ pureza/degradación $(\alpha=.84)$, y un índice Alfa de Cronbach global $=.84$. También muestra índices de validez convergente y divergente con base en correlaciones de Pearson con escalas de empatía, valores y actitudes hacia diferentes temas como religión o política. No se reporta la varianza explicada.

\section{Procedimiento}

Se realizó un procedimiento de aplicación igual a los estudios 1 y 2 . Los datos se capturaron con ayuda del programa SPSS versión 24 y se analizaron con el programa AMOS, en el que se obtuvieron los resultados.

\section{Resultados}

Se realizó un análisis factorial confirmatorio con el método de Máxima Verosimilitud. Se consideraron los índices $\chi^{2} / \mathrm{gl}$ con valores esperados menores a 3, GFI (Goodness of Fit Index) con valores esperados mayores a $.95 \mathrm{y}$ mayores a .90 como aceptables, SRMR (Standardized Root of Mean Squared Residual) con valores esperados menores a .08 y RMSEA (Root Mean Square Error of Approximation) con valores esperados menores a .08 como aceptables.

Se mantuvo el modelo de cinco factores con sus respectivos índices de ajuste $\left(\chi^{2}=432.244, \mathrm{gl}=160\right.$, 


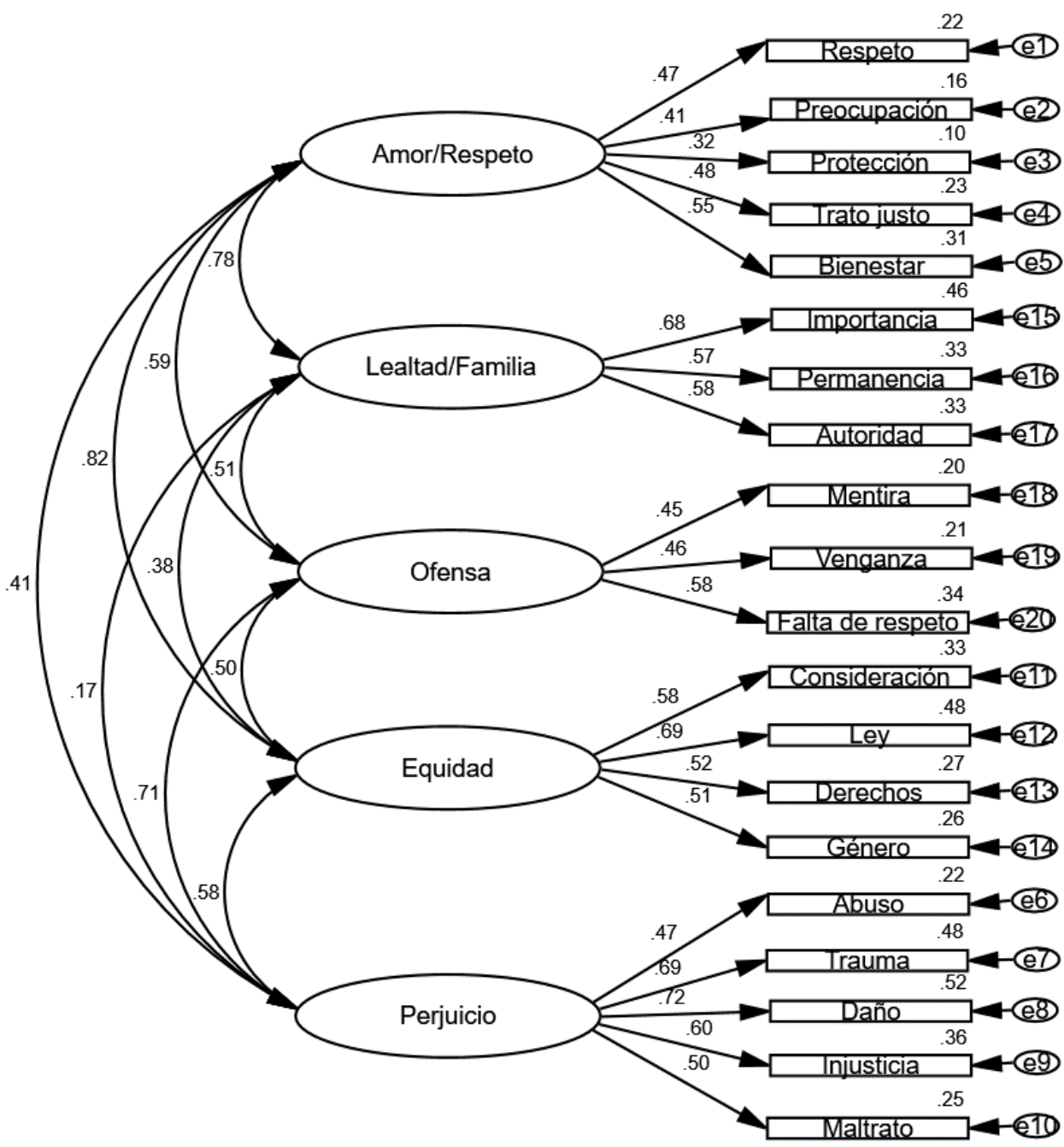

Figura 1. Estructura factorial del dominio moral en adolescentes $(\mathrm{N}=410)$

Tabla 5. Correlaciones de los factores de la Escala de Dominio Moral para Adolescentes y el Cuestionario de los Fundamentos Morales $(\mathrm{N}=410)$

\begin{tabular}{cccccc}
\hline \multirow{2}{*}{$\begin{array}{c}\text { Factores dominio moral } \\
\text { adolescentes }\end{array}$} & $\begin{array}{c}\text { Cuidado/ } \\
\text { daño }\end{array}$ & $\begin{array}{c}\text { Justicia/ } \\
\text { injusticia }\end{array}$ & $\begin{array}{c}\text { Lealtad/ } \\
\text { traición }\end{array}$ & $\begin{array}{c}\text { Autoridad/ } \\
\text { subversión }\end{array}$ & $\begin{array}{c}\text { Pureza/ } \\
\text { degradación }\end{array}$ \\
\cline { 2 - 5 } & $.240^{* *}$ & $.150^{* *}$ & $.230^{* *}$ & $.247^{* *}$ & $.177^{* *}$ \\
Amor/respeto & $.101^{*}$ & .059 & $.272^{*}$ & $.242^{* *}$ & $.237^{* *}$ \\
Lealtad/familia & $.256^{* *}$ & $.209^{* *}$ & $.179^{* *}$ & $.193^{* *}$ & $.218^{* *}$ \\
Ofensa & $.252^{*}$ & $.242^{* *}$ & $.109^{*}$ & $.133^{* *}$ & .021 \\
Equidad & $.312^{* *}$ & $.319^{* *}$ & $.117^{*}$ & $.103^{*}$ & .077 \\
Perjuicio & .01. & & &
\end{tabular}

** Correlación estadísticamente significativa al .01.

* Correlación estadísticamente significativa al .05.

$p<.001, \quad \chi^{2} / \mathrm{gl}=2.701, \quad \mathrm{GFI}=.902, \quad \mathrm{SRMR}=.063$, RMSEA $=.064$ ), donde los índices GFI y RMSEA se consideran aceptables (Ferrando \& AnguianoCarrasco, 2010) y los índices $\chi^{2} / g 1$ y SRMR indican que se presenta un buen ajuste del modelo a los datos (Abad et al., 2011). En la Figura 1 se muestra la estructura factorial de la escala.

Posteriormente al Análisis Factorial Confirmatorio, se identificó la relación de los principios morales en adolescentes con los propuestos por la teoría de los fundamentos morales a través de correlaciones productomomento de Pearson. En la Tabla 5 se muestra la matriz de correlaciones.

\section{Discusión}

Se elaboró una escala de dominio moral para adolescentes que integra cinco factores: 
amor/respeto, lealtad/familia, ofensa, equidad y perjuicio. Es un instrumento que aporta conocimiento empírico sobre el tema y que puede apoyar a futuras investigaciones. Se menciona que la estructura del dominio moral favorece la promoción de principios morales relevantes para los adolescentes que los motive a ta participar en la resolución de problemas sociales (Conde-Flores et al., 2017; Kollerová et al., 2014). Se estima que la conceptuación de dominio moral, así como sus componentes ofrecen elementos que pueden facilitar estas necesidades, debido a que los principios morales propuestos tienen una estrecha relación con estudios de la moralidad en general y con la cultura mexicana en particular.

El principio moral de amor y respeto corresponde con la mención de Haidt (2006), acerca del amor como un sistema de cuidados y vínculos afectivos donde se da valor a los seres queridos. De igual manera, en la cultura mexicana el amor predomina como eje del comportamiento de las personas y el respeto se caracteriza por su aceptación y valoración como seres humanos, por acciones de amar y sentir afecto por alguien, así como dar y recibir protección (Díaz-Guerrero, 1994, 2003). A pesar de tener elementos sobre el cuidado, este principio se muestra conceptualmente diferente de los principios mencionados en la teoría de los fundamentos morales (Graham et al., 2013). Su planteamiento se fundamenta en una ética del sufrimiento o del daño para reconocer la importancia de cuidar como una acción moral. Se estima que la diferencia se debe a la separación de conceptos con valores positivos y negativos (Wiltermuth et al., 2010), por lo que se sugiere mantener esta separación al momento de estudiar aspectos relacionados con la moralidad.

El principio de lealtad/familia se adecua a las investigaciones en la cultura mexicana donde el honor familiar, el respeto a los padres y los intereses del entorno familiar antes que los del individuo, forman parte de las principales premisas del comportamiento del mexicano (DíazGuerrero, 1994, 2003). A su vez, en la cultura mexicana, la lealtad es un componente importante en las normas y valores que intervienen en un funcionamiento familiar positivo (García-Méndez, Rivera-Aragón, Reyes-Lagunes, \& Díaz-Loving, 2006).
El principio de ofensa sugiere que las personas infractoras son juzgadas por acciones en las que se percibe un fallo como persona (Chakroff, Dungan, \& Young, 2013). En general, puede haber una expresión de sentimientos de disgusto y de desprecio debido a las acciones que implican falta de honor, por lo que es probable un distanciamiento con la persona infractora (Haidt, 2003, 2006).

En el caso de la ofensa, a diferencia del daño o el perjuicio, se caracteriza por una falta a los valores y principios morales sin la presencia de una afección física o psicológica directa y que se puede transmitir en términos de perdonar la acción o expresar resentimiento en contextos específicos (Rosales-Sarabia, Rivera-Aragón, Reidl-Martínez, \& García-Méndez, 2018; Worthington, 2006).

El principio de equidad favorece la reciprocidad en las relaciones interpersonales con fines de bienestar social, representa un acuerdo de hombres y mujeres con las normas de justicia y responsabilidad mutua, en condiciones de igualdad y cooperación con las leyes de la sociedad (Díaz-Loving et al., 2015; Graham et al., 2013; Haidt \& Joseph, 2004, 2007).

El principio de perjuicio considera una moralidad diádica, donde existe un agresor o agente y una persona afectada o víctima (Gray, Young, \& Waytz, 2012). Los sentimientos que promueven estos juicios suelen ser el enojo y el deseo de sanción para el agente y la compasión o consideración por la persona afectada (Chakroff et al., 2013). El perjuicio se asume como la acción de afectar a otros, se percibe como un trato injusto que se debe evitar (Graham et al., 2011). Se rechaza la violación a los derechos de justicia y bienestar, que corresponden a una ética de la autonomía y la integridad (Kwan, 2016).

La lealtad a la familia, la ofensa, la equidad y el perjuicio muestran similitudes con estudios previos relacionados con el sufrimiento, la compasión por los vulnerables, la búsqueda de relaciones justas, el valor a los grupos de pertenencia y los sentimientos de disgusto ante estímulos desagradables o que contaminen estándares de pureza (Haidt \& Joseph, 2004, 2007). Sin embargo, existen diferencias en cuanto al contenido conceptual de cada principio, debido a las particularidades culturales que están 
presentes en la expresión de la moralidad (Haste \& Abrahams, 2008).

Los cinco principios morales identificados en este estudio marcan una clasificación de patrones de comportamiento y socialización, por lo que propone identificar cómo estos principios pueden ser diferentes en otros contextos sociales de la república mexicana y en otras culturas. Al respecto, Haste (2013), refiere que es importante dar sentido a los componentes del dominio moral a partir de su relación con el contexto cultural e histórico al que las personas están expuestas, con la finalidad de proponer normas y prácticas cívicas y comunitarias que sean accesibles para la sociedad a la que pertenecen.

Los principios morales resultado de esta investigación muestran similitudes y diferencias con los principios identificados en la teoría de los fundamentos morales, lo que sugiere que la interacción con la cultura, la experiencia y los procesos de socialización, modifican el entendimiento de estos fundamentos. Se sugiere que las similitudes y diferencias se deben a las bases intuitivas e innatas de la moralidad y a la influencia de la cultura, es decir, a los cambios que moldean la estructura y el contenido de estos fundamentos (Haste, 2013).

La noción del dominio moral durante la adolescencia es un proceso susceptible a modificarse en la etapa adulta, tal como sugiere Hauser (2006) quien menciona que en la adultez se consolidan los principios morales de la cultura.

La propuesta de estructura del dominio moral de esta investigación se enfoca en los conceptos que se reconocen durante la adolescencia, más que en sus intuiciones que son la base teórica de los fundamentos morales. Se destaca esta diferencia, debido a que la perspectiva de la intuición resalta la importancia de probar la naturaleza innata de un principio moral para que se reconozca como un fundamento moral (Graham et al., 2013). Sin embargo, al tratarse de los conceptos utilizados en el discurso de los adolescentes, es posible acercarse a la forma en que piensan y se motivan para la participación cívica y la acción moral (Haste \& Abrahams, 2008).

Con la finalidad de ampliar y fortalecer el conocimiento sobre el tema de estudio, se sugiere realizar otras investigaciones que se remitan a los orígenes del juicio moral para reconocer la naturaleza intuitiva de los principios identificados en esta investigación. De igual manera es importante indagar sobre evaluación de la moral a partir de diferentes culturas en diferentes etapas del desarrollo, con el objetivo de proponer elementos de educación moral relevantes para la población meta de cada investigación.

En general, se considera conveniente realizar estudios con el enfoque de prevención de conductas antisociales, en los que se identifique cómo se expresa la moralidad en adolescentes infractores cuando se les pregunta acerca de principios morales propios de su etapa del desarrollo, con la finalidad de reducir índices de conductas indeseables presentes en la adolescencia a través de la educación basada en principios morales relevantes.

\section{Agradecimientos}

Se agradece al Consejo Nacional de Ciencia y Tecnología (CONACYT) por la beca otorgada al primer autor con número de becario 609829 y CVU 779456, con la que se financió la presente investigación.

\section{Referencias}

Abad, F. J., Olea, J., Ponsoda, J., \& García, C. (2011). Medición en ciencias sociales y de la salud. Madrid: Síntesis.

Aguilar, J., Valencia, A., \& Sarmiento, C. (2007). Relaciones familiares y ajuste personal, escolar y social en la adolescencia. México: Universidad Nacional Autónoma de México.

Barrios, A., Barbato, S., \& Branco, A. (2012). El análisis microgenético para el estudio del desarrollo moral: Consideraciones teóricas y metodológicas. Revista de Psicología, 30(2), 249-279.

Bedregal, P., León, T., Shand, B., \& Mosso, L. (2013). Criterios de juicio moral de estudiantes de Medicina, según el Modelo Intuitivo Social. Revista médica de Chile, 141(6), 704-709.

doi:10.4067/S0034-98872013000600003.xs

Berniūnas, R., Dranseika, V., \& Sousa, P. (2016). Are there different moral domains? Evidence from Mongolia. Asian Journal of Social 
Psychology,

19(3),

275-282.

doi:10.1111/ajsp.12133.

Chakroff, A., Dungan, J., \& Young, L. (2013). Harming ourselves and defiling others: What determines a moral domain? PloS one, 8(9), e74434. doi:10.1371/journal.pone.0074434.

Clifford, S., Iyengar, V., Cabeza, R., \& SinnottAmstrong, W. (2015). Moral foundations vignettes: A standarized stimulus database of scenarios based on foundations theory. Behavior Research Methods, 47(4). 11781198. doi:10.3758/s13428-014-0551-2.

Conde-Flores, S., García-Cabrero, B., \& AlbaMeraz, A. (2017). Civic and ethical education in Mexico. In B. García-Cabrero, A. Sandoval-Hernández, E. Treviño-Villareal, S., Ferráns, \& M., Martínez (Eds.), Civics and Citizenship: Theoretical Models and Experiences in Latin America (pp. 41-66). The Netherlands: Sense Publishers.

De Waal, F. (1996). Good Natured-The origins of right \& wrong in humans and other animals. U.S.A.: Harvard University Press.

Díaz-Guerrero, R. (1994). Psicología del mexicano. México: Editorial Trillas.

Díaz-Guerrero, R. (2003). Bajo las garras de la cultura: Psicología del mexicano 2. México: Editorial Trillas.

Díaz-Loving, R. (2011). Dinámica de las premisas histórico-socio-culturales: Trayecto, vigencia y prospectiva. Revista Mexicana de Investigación en Psicología, 3(2), 174-180.

Díaz-Loving, R., Saldívar, A., Armenta-Hurtarte, C., Reyes, N. E., López, F., Moreno, M., ... \& Correa, F. E. (2015). Creencias y normas en México: Una actualización del estudio de las premisas psico-socio-culturales. Psykhe (Santiago), $\quad 24(2), \quad 1-25$, doi:10.7764/psykhe.24.2.880.

Doyle, F. L., \& Bussey, K. (2018). Moral disengagement and children's propensity to tell coached lies. Journal of Moral Education, 47(1), 91-103. doi:10.1080/03057240.2017.1380611.

Ferrando, P. J., \& Anguiano-Carrasco, C. (2010). El análisis factorial como técnica de investigación en psicología. Papeles del psicólogo, 31(1), 18-33.

Garaigordobil, M. (2005). Conducta antisocial durante la adolescencia: Correlatos socioemocionales, predictores y diferencias de género. Psicología conductual, 13(12), $197-$ 215.

García-Méndez, M., Rivera-Aragón. S., ReyesLagunes, I., \& Díaz-Loving, R. (2006). Construcción de una escala de funcionamiento familiar. Revista Iberoamericana de Diagnóstico y Evaluación Psicológica - e Avaliação Psicológica. 2(22), 91-110.

Gibbs, J. C., Arnold, K. D., Morgan, R. L., Schwartz, E. S., Gavaghan, M. P., \& Tappan, M. B. (1984). Construction and validation of a multiple-choice measure of moral reasoning. Child Development, 55(2). 527536.

Góngora, V. C., \& Castro-Solano, A. (2014). Well-being and life satisfaction in Argentinean adolescents. Journal of Youth Studies, 17(9), 1277-1291. doi:10.1080/13676261.2014.918251.

Graham, J., Haidt, J., Koleva, S., Motyl, M., Iyer, R., Wojcik, S., \& Ditto, P. (2013). Moral foundations theory: The pragmatic validity of moral pluralism. Advances in Experimental Social Psychology, 47(2013), 55-130. doi:10.1016/B978-0-12-407236-7.00002-4.

Graham, J., Nosek, B., Haidt, J., Iyer, R., Koleva, S., \& Ditto, P. (2011). Mapping the moral domain. Journal of Personality and Social Psychology, 101(2), 366-385. doi:10.1037/a0021847.

Gray, K., Young, L., \& Waytz, A. (2012). Mind perception is the essence of morality. Psychological inquiry, 23(2), 101-124. doi:10.1080/1047840X.2012.651387.

Haidt, J. (2003). The moral emotions. En R. J. Davidson, K. R. Scherer \& H. H. Goldsmith (Eds.), Handbook of affective sciences. Oxford: Oxford University Press. 852-870.

Haidt, J. (2006). La hipótesis de la felicidad. España: Gedisa.

Haidt, J., \& Joseph, C. (2004). Intuitive ethics: How innately prepared intuitions generate culturally variable virtues. Daedalus, 133(4), 55-66. doi:10.1162/0011526042365555.

Haidt, J. \& Joseph, C. (2007). The moral mind: How 5 sets of innate moral intuitions guide the development of many culture-specific virtues, and perhaps even modules. In P. Carruthers, S. Laurence, \& S. Stich (Eds.) The 
Innate Mind, Vol. 3. New York: Oxford. 367391.

Harré, R. (2002). Material objects in social worlds. Theory, Culture \& Society, 19(5-6), 23- 33. doi: 10.1177/026327640201900502.

Haste, H. (2013). Deconstructing the elephant and the flag in the lavatory: Promises and problems of moral foundations research. Journal of Moral Education, 42(3), 316-329. doi: 10.1080/03057240.2013.818529.

Haste, H., \& Abrahams, S. (2008). Morality, culture and the dialogic self: Taking cultural pluralism seriously. Journal of Moral Education, 37(3), 377-394. doi:10.1080/03057240802227502.

Haste, H., Bermúdez, A., \& Carretero, M. (2017). Culture and civic competence. In B., GarcíaCabrero, A., Sandoval-Hernández, E., Treviño-Villareal, S., Ferráns, \& M., Martínez (Eds.). Civics and Citizenship: Theoretical Models and Experiences in Latin America. The Netherlands: Sense Publishers. 3-15.

Hauser, M. (2006). La mente moral. Cómo la naturaleza ha desarrollado nuestro sentido del bien y del mal. España: Paidós.

Kohlberg, L. (1987). The development of moral judgment and moral action. In L. Kohlberg (Ed.). Child psychology and childhood education. A cognitive-developmental view. New York: Longman. 259-328.

Kollerová, L., Janošová, P., \& Říčan, P. (2014). Good and evil at school: Bullying and moral evaluation in early adolescence. Journal of Moral Education, 43(1), 18-31. doi:10.1080/03057240.2013.866940.

Kwan, L. (2016). Anger and perception of unfairness and harm: Cultural differences in normative processes that justify sanction assignment. Asian Journal of Social Psychology, 19(1), 6-15. doi: 10.1111/ajsp.12119.

Kwok, J., \& Selman, R. (2017). From informed social reflection to civic engagement. In B., García-Cabrero, A., Sandoval-Hernández, E., Treviño-Villareal, S., Ferráns, \& M., Martínez (Eds.). Civics and Citizenship: Theoretical Models and Experiences in Latin America. The Netherlands: Sense Publishers. 17-38.

Leenders, I., \& Brugman, D. (2005). Moral/nonmoral domain shift in young adolescents in relation to delinquent behaviour. British Journal of Developmental Psychology, 23(1), 65-79. doi:10.1348/026151004X20676.

Lees, J., \& Gino, F. (2017). Is the moral domain unique? A social influence perspective for the study of moral cognition. Social and Personality Psychology Compass, 11(8), 123127. doi:10.1111/spc3.12327.

Mansilla, M. (2000). Etapas del desarrollo humano. Revista de investigación en psicología, 3, 105-116.

Mayring, P. (2014). Qualitative Content Analysis: Theoretical Foundation, Basic Procedures and Software Solution. Klagenfurt: Open Access Repository.

Palmer, E. J., \& Hollin, C. R. (1998). A comparison of patterns of moral development in young offenders and non- offenders. Legal and Criminological Psychology, 3(2), 225235.doi: 10.1111/j.2044-8333.1998.tb00363.x

Pinker, S. (2002). The blank slate. The modern denial of human nature. New York, NY: Viking.

Pozzoli, T., Gini, G., \& Thornberg, R. (2016). Bullying and defending behavior: The role of explicit and implicit moral cognition. Journal of school psychology, 59, 67-81. doi: 10.1016/j.jsp.2016.09.005.

Railton, P. (1986). Moral realism. The Philosophical Review, 95(2), 163-207.

Reinserta Un Mexicano, A. C. (2018). Estudio de factores de riesgo y victimización en adolescentes que cometieron delitos de alto impacto social. Recuperado del sitio de Internet de Reinserta Un Mexicano A.C: https://reinserta.org/

Rosales-Sarabia, R., Rivera-Aragón, S., ReidlMartínez, L., \& García-Méndez, M. (2018). Perdón y Resentimiento hacia la pareja: Desarrollo y validación de dos escalas. Revista Iberoamericana de Diagnóstico y Evaluación - e Avaliação Psicológica 2(47), 141-156. doi: 10.21865/RIDEP47.2.10.

Sanabria, A., \& Uribe, A. (2009). Conductas antisociales y delictivas en adolescentes infractores y no infractores. Pensamiento psicológico, 6(13), 203-218.

Shweder, R., Mahapatra, M., \& Miller, J. (1987). Culture and moral development. In J. Kagan and S. Lamb. (Eds.). The emergence of 
morality in young children. Chicago:

University of Chicago Press. 1-83.

Turiel, E. (1983). The development of social knowledge: Morality and convention. United Kingdom: Cambridge University Press.

Wiltermuth, S. S., Monin, B., \& Chow, R. M. (2010). The orthogonality of praise and condemnation in moral judgment. Social Psychological and Personality Science, 1(4), 302-310. doi:10.1177/1948550610363162.

Wojciszke, B. (2005). Morality and competence in person-and self-perception. European review of social psychology, 16(1), 155-188. doi:10.1080/10463280500229619.

Worthington, E. (2006). Forgiveness and reconciliation: Theory and application. New York: Routledge. 


\section{ANEXO 1}

CUESTIONARIO DE PREGUNTAS ABIERTAS SOBRE DOMINIO MORAL EN ADOLESCENTES

1. ¿Cuál es la importancia que consideras tiene el cuidado hacia los demás o hacia uno mismo?

2. ¿Qué implicaciones crees que tiene hacerles daño a las personas o a uno mismo?

3. En tu experiencia, ¿qué acciones consideras que son justas?

4. En tu experiencia, ¿qué acciones consideras que son injustas?

5. Para ti, ¿con qué tiene que ver la lealtad?

6. Para ti, ¿con qué tiene que ver la traición?

7. ¿Cómo crees que debe ser una persona con autoridad razonable?

8. ¿Qué consideras que justifica el hecho de que las personas se rebelen ante una autoridad?

9. Para ti, ¿qué es una persona pura?

10. ¿Qué cosas consideras que degradan la pureza de las personas?

11. Para ti, ¿con qué se relaciona la moralidad?

12. Para ti, ¿con qué se relaciona lo inmoral? 


\section{ANEXO 2}

\section{ESCALA DE DOMINIO MORAL PARA ADOLESCENTES}

A continuación, se presenta una serie de afirmaciones sobre lo que está bien y lo que está mal, por favor señala qué tan de acuerdo o en desacuerdo estás con ellas.

1. Muy en desacuerdo

2. Moderadamente en desacuerdo

3. Levemente en desacuerdo

4. Levemente de acuerdo

5. Moderadamente de acuerdo

6. Muy de acuerdo

\begin{tabular}{|c|c|c|c|c|c|c|c|}
\hline 1 & La mentira es un camino que sólo lleva a malas consecuencias. & 1 & 2 & 3 & 4 & 5 & 6 \\
\hline 2 & Es indignante que algunas personas se beneficien a costa del esfuerzo de otros. & 1 & 2 & 3 & 4 & 5 & 6 \\
\hline 3 & Todos tenemos derecho a que nuestra opinión sea tomada en cuenta. & 1 & 2 & 3 & 4 & 5 & 6 \\
\hline 4 & La lealtad a la familia es lo más importante de todo. & 1 & 2 & 3 & 4 & 5 & 6 \\
\hline 5 & El respeto es el camino necesario para ser una mejor sociedad. & 1 & 2 & 3 & 4 & 5 & 6 \\
\hline 6 & La venganza es la peor forma de buscar justicia. & 1 & 2 & 3 & 4 & 5 & 6 \\
\hline 7 & El respeto es necesario para jurar lealtad a los seres queridos. & 1 & 2 & 3 & 4 & 5 & 6 \\
\hline 8 & Es necesaria una autoridad que se preocupe por el bienestar de todos. & 1 & 2 & 3 & 4 & 5 & 6 \\
\hline 9 & Es horrible que una persona le provoque traumas emocionales a otra. & 1 & 2 & 3 & 4 & 5 & 6 \\
\hline 10 & La ley se debe aplicar a todos por igual, sin importar si son ricos o pobres. & 1 & 2 & 3 & 4 & 5 & 6 \\
\hline 11 & Pienso que es inaceptable dañar a otras personas. & 1 & 2 & 3 & 4 & 5 & 6 \\
\hline 12 & La falta de respeto sólo lleva a la gente a malas consecuencias. & 1 & 2 & 3 & 4 & 5 & 6 \\
\hline 13 & Cuidar a otras personas es una manera de expresar lo valiosas que son. & 1 & 2 & 3 & 4 & 5 & 6 \\
\hline 14 & Las personas siempre deben ser leales a los miembros de su familia. & 1 & 2 & 3 & 4 & 5 & 6 \\
\hline 15 & $\begin{array}{l}\text { Todas las personas tienen los mismos derechos, sin importar su preferencia } \\
\text { sexual. }\end{array}$ & 1 & 2 & 3 & 4 & 5 & 6 \\
\hline 16 & Tratar a las personas con respeto ayuda a ser una sociedad más justa. & 1 & 2 & 3 & 4 & 5 & 6 \\
\hline 17 & Procurar el bienestar de aquellos que amamos es lo más importante. & 1 & 2 & 3 & 4 & 5 & 6 \\
\hline 18 & $\begin{array}{l}\text { Es injusto que una sola persona se quede todo el crédito de un logro que se obtuvo } \\
\text { en equipo. }\end{array}$ & 1 & 2 & 3 & 4 & 5 & 6 \\
\hline 19 & La equidad de género es un valor indispensable. & 1 & 2 & 3 & 4 & 5 & 6 \\
\hline 20 & Una persona que le guste perjudicar a los demás es un ser desagradable. & 1 & 2 & 3 & 4 & 5 & 6 \\
\hline
\end{tabular}

